\title{
Réadaptation hospitalière: forfaits journaliers liés aux prestations
}

\author{
Petra Ingenpass a , Barbara Rohner \\ ${ }^{a}$ Dr, cheffe adjointe de la division Tarifs et économie de la santé pour les médecins hospitaliers; ${ }^{b}$ collaboratrice scientifique
} living. Cela comprend l'EBI (indice de Barthel étendu), le FIM = Functional Independence Measure et le $\mathrm{HAQ}=$ tionnaire (jusqu'à fin 2015.)

2 CIRS = Cumulative Illness Rating Scale médicales, infirmières et thérapeutiques

4 Par ex. coûts d'hôtellerie, d'infrastructure et d'administration Publications $\rightarrow$ Publications techniques $\rightarrow$ Document de référence DefReha ${ }^{\bullet}$ Version 1.0
$1 \mathrm{ADL}=$ Activities of daily Health Assessment Ques-

3 Coûts des prestations

5 www.hplus.ch/fr $\rightarrow$

La réadaptation hospitalière doit à l'avenir être indemnisée dans toute la Suisse par des forfaits journaliers liés aux prestations. La rémunération dépendra ainsi du degré de gravité de la limitation fonctionnelle du patient ainsi que de l'intensité du traitement. Il s'agit là d'une nette amélioration que la FMH soutient résolument, mais elle estime que d'autres mesures sont nécessaires.

\begin{abstract}
A l'heure actuelle, la réadaptation hospitalière est le plus souvent rémunérée par des forfaits journaliers uniformes. Or la loi sur l'assurance-maladie (LAMal) prévoit des forfaits liés aux prestations pour les traitements hospitaliers. De ce fait, des forfaits journaliers nationaux uniformes liés aux prestations sont en cours de développement pour une structure tarifaire de la réadaptation hospitalière. Appelée ST Reha, cette nouvelle structure tarifaire doit être simple, vérifiable et extensible, et contenir le moins possible d'incitatifs erronés. Aucun tarif comparable n'existant pour l'instant à l'étranger, ST Reha 0.2 livre pour la première fois une version préliminaire de la structure tarifaire pour la réadaptation neurologique et musculo-squelettique.
\end{abstract}

\section{Quatre domaines de prestations}

Les points essentiels de développement de la structure tarifaire pour la réadaptation hospitalière résident dans la nature du traitement et le degré de gravité de la limitation fonctionnelle. N'ayant aucune valeur prédictive, les diagnostics ne jouent aucun rôle. Un algorithme conçu avec une série de questions claires classe le patient en fonction de ses troubles fonctionnels dans un des quatre domaines de prestations définis. Le degré de gravité de la limitation fonctionnelle est
Structure tarifaire pour la réadaptation hospitalière

(ST Reha)

Les exigences légales en matière de forfaits liés aux prestations impliquent les change-

ments suivants pour les tarifs hospitaliers:

des forfaits journaliers échelonnés en fonction des prestations effectuées

- une définition de quatre domaines de prestations: neurologique, cardio-vasculaire, pulmonaire et autres (par ex. musculo-squelettique)

- des évaluations nationales uniformes pour déterminer le degré de gravité de la limitation fonctionnelle

Introduction de la nouvelle structure tarifaire en 2018. obtenu pour chaque domaine de prestations sur la base d'évaluations (tabl. 1). Le score ADL, qui mesure l'autonomie du patient au quotidien [1], a une valeur probante élevée.

\section{Une rémunération plus adéquate grâce à un tarif lié aux prestations}

Pour la validation des systèmes de classification des patients en réadaptation neurologique et musculosquelettique, dix cliniques ont fourni en 2013 leurs données sur les prestations et les coûts à la Haute école zurichoise de sciences appliquées (ZHAW), chargée du développement de la structure tarifaire. En plus des données administratives et des coûts variables [3] liés aux patients, les coûts fixes [4] ont également été pris en compte. Dans la version préliminaire de la structure tarifaire pour les domaines neurologique et musculo-squelettique, la ZHAW a proposé sur cette base un petit nombre de groupes tarifaires différents, avec les coûts relatifs correspondants; les affectations en réadaptation cardiaque ou pulmonaire seront évaluées plus tard. Les coûts relatifs multipliés par le taux de base (baserate), négocié préalablement par les partenaires, donnent ainsi les forfaits journaliers pour la réadaptation. Selon le document DefReha ${ }^{\circledR} \mathrm{de}$ $\mathrm{H}+$ [5], les données 2015 permettront également des évaluations complémentaires concernant les domaines de réadaptation. Pour la FMH, il est cependant important que les domaines de la réadaptation soient représentés de manière appropriée dans la nouvelle structure tarifaire; d'autres domaines de prestations seraient éventuellement à étudier. Par ailleurs, il faut s'atteler sans délai à la définition stricte de ce qu'est un cas de réadaptation en précisant la frontière avec les soins somatiques aigus et la psychiatrie, et procé- 
Tableau 1: Evaluations de la réadaptation hospitalière.

\begin{tabular}{llll} 
Neurologique & Cardio-vasculaire & Pulmonaire & Autre \\
\hline CIRS [2] & CIRS & - & CIRS \\
\hline ADL & ADL & ADL & ADL \\
\hline- & - & Test de marche de 6 min. & - \\
\hline- & - & - & Douleur \\
\hline
\end{tabular}

der à une classification claire des patients en «réadaptation précoce» [6]. Enfin, la version tarifaire ST Reha 0.2 proposée actuellement ne tient pas encore compte des prestations autres que celles du domaine de la réadaptation. Celles-ci sont saisies et évaluées dans le cadre d'une collecte de données complémentaire; des travaux sont en cours pour trouver des solutions de rémunération appropriée.

\section{Potentiel d'amélioration de la qualité des données}

Le développement de la structure tarifaire ST Reha version 0.2 pour la réadaptation neurologique et musculo-squelettique repose sur les données de 2013 provenant de toutes les régions linguistiques. Les prestations médicales, infirmières et thérapeutiques ont été associées aux diagnostics, aux données administratives et aux résultats des évaluations hebdomadaires. Après exclusion des données de patients présentant des indications insuffisamment détaillées ou imprécises, deux tiers des données récoltées ont été utilisées pour le développement de la structure tarifaire. L'objectif est de parvenir à un système de rémunération qui réponde mieux aux moyens investis en se fondant sur des coûts relevés le plus précisément possible pour chaque cas particulier.

De ce fait, il est primordial que le plus grand nombre possible de cliniques fournissent des données complètes de bonne qualité pour la collecte des données 2015; d'une part parce que la version d'introduction prévue pour 2018 sera développée sur la base de données collectées cette année; et d'autre part, parce que ces données permettront de vérifier la représentativité des domaines figurant sous "Autre réadaptation». S'il serait pertinent de réfléchir à une certification selon REKOLE $^{\circledR}$, SwissDRG SA doit de toute façon élaborer un concept en vue d'améliorer la qualité des données. La saisie des troubles fonctionnels nécessitant une réadaptation dépend des évaluations hebdomadaires, sans qu'aucune procédure uniforme n'existe. Il s'agit ici de réduire les marges d'appréciation par des définitions contraignantes et des directives précises. Une bonne qualité des données n'est possible que si l'utilisation des instruments de mesure nécessaires et la saisie des données de coûts sont uniformes.

\section{L'attribution aux domaines de prestations relève du médecin}

Pour l'instant, quatre domaines de prestations sont définis pour la réadaptation hospitalière. Selon la FMH, c'est au médecin traitant et à lui seul qu'il appartient de décider du domaine où classer le patient nécessitant une réadaptation; il doit le faire sur la base du trouble fonctionnel principal existant. Au moment de l'octroi de la garantie de prise en charge des coûts de la réadaptation hospitalière, le risque existe cependant qu'une réadaptation moins chère soit préférée à celle indiquée par le médecin. Il faut donc veiller à ce que le traitement de réadaptation corresponde aux besoins du patient.

\section{Nécessité d'une réglementation transitoire et d'une recherche concomitante}

Comme aucune structure tarifaire n'a encore été établie dans aucun pays, aucune donnée de référence ne précède le développement de ST Reha. Ce n'est donc qu'avec la version d'introduction prévue pour 2018 qu'on disposera d'une structure tenant compte pour la première fois de tous les domaines de réadaptation concernés, et ainsi, par exemple, de la réadaptation gériatrique. Pour l'heure, personne ne sait comment les grandes différences régionales en matière de conception des soins seront prises en considération. Les cliniques n'auront donc pas la possibilité de se familiariser en détail avec la structure tarifaire avant son introduction. C'est pourquoi la FMH rappelle sa demande d'introduction d'une réglementation transitoire, déjà formulée dans sa prise de position de 2012 [7]. Par ailleurs, l'impossibilité d'une comparaison internationale accentue la nécessité de mettre en place suffisamment tôt une recherche concomitante pour évaluer la situation avant et après l'introduction de ST Reha.

\section{Conclusion et perspectives}

La FMH soutient expressément la voie choisie, car elle mène à une rémunération plus adéquate des prestations médicales. Diverses questions restent cependant encore ouvertes et il faudra y répondre dans un délai ambitieux. Pour la FMH, les points suivants sont à traiter en priorité: souveraineté décisionnelle du médecin, développement de forfaits journaliers sur la base de données de bonne qualité, réglementation transitoire et recherche concomitante. Afin que les différents besoins des médecins du pays puissent être intégrés dans la suite du développement de la structure tarifaire, les représentants de toutes les régions sont invités à transmettre leurs avis au groupe de suivi ST Reha de la FMH via les sociétés de discipline médicale concernées. 